\title{
Interactions Between Drought and Plant Genotype Change Epidemiological Traits of Cauliflower mosaic virus
}

\author{
Sandy E. Bergès ${ }^{1,2}$, Denis Vile ${ }^{2 *}$, Cecilia Vazquez-Rovere ${ }^{2,3}$, Stéphane Blanc ${ }^{1}$, \\ Michel Yvon ${ }^{1}$, Alexis Bédiée ${ }^{2}$, Gaëlle Rolland ${ }^{2}$, Myriam Dauzat ${ }^{2}$ and \\ Manuella van Munster ${ }^{1 \star}$
}

' BGPI, CIRAD, INRA, Montpellier SupAgro, Université de Montpellier, Montpellier, France, ${ }^{2}$ LEPSE, INRA, Montpellier SupAgro, Université de Montpellier, Montpellier, France, ${ }^{3}$ LABINTEX Europe, Instituto Nacional de Tecnología Agropecuária, Montpellier, France

OPEN ACCESS

Edited by:

Muthappa Senthil-Kumar, National Institute of Plant Genome Research (NIPGR), India

Reviewed by: Kazuo Nakashima Japan International Research Center for Agricultural Sciences, Japan Aarti Gupta,

National Institute of Plant Genome Research (NIPGR), India Nobuhiro Suzuki,

Sophia University, Japan

*Correspondence: Denis Vile denis.vile@inra.fr

Manuella van Munster manuella.van-munster@inra.fr

Specialty section: This article was submitted to Plant Abiotic Stress,

a section of the journal Frontiers in Plant Science

Received: 16 February 2018 Accepted: 08 May 2018

Published: 24 May 2018

Citation:

Bergès SE, Vile $D$ Vazquez-Rovere $C$, Blanc S, Yvon $M$, Bédiée A, Rolland G, Dauzat $M$ and van Munster M (2018) Interactions Between Drought and Plant Genotype Change Epidemiological Traits of Cauliflower mosaic virus.

Front. Plant Sci. 9:703. doi: 10.3389/fpls.2018.00703
Plants suffer from a broad range of abiotic and biotic stresses that do not occur in isolation but often simultaneously. Productivity of natural and agricultural systems is frequently constrained by water limitation, and the frequency and duration of drought periods will likely increase due to global climate change. In addition, phytoviruses represent highly prevalent biotic threat in wild and cultivated plant species. Several hints support a modification of epidemiological parameters of plant viruses in response to environmental changes but a clear quantification of plant-virus interactions under abiotic stresses is still lacking. Here we report the effects of a water deficit on epidemiological parameters of Cauliflower mosaic virus (CaMV), a non-circulative virus transmitted by aphid vectors, in nine natural accessions of Arabidopsis thaliana with known contrasted responses to water deficit. Plant growth-related traits and virus epidemiological parameters were evaluated in PHENOPSIS, an automated high throughput phenotyping platform. Water deficit had contrasted effects on CaMV transmission rate and viral load among $A$. thaliana accessions. Under well-watered conditions, transmission rate tended to increase with viral load and with CaMV virulence across accessions. Under water deficit, transmission rate and virulence were negatively correlated. Changes in the rate of transmission under water deficit were not related to changes in viral load. Our results support the idea that optimal virulence of a given virus, as hypothesized under the transmission-virulence trade-off, is highly dependent on the environment and growth traits of the host.

Keywords: plant growth traits, viral load, viral transmission, virulence, tolerance, plant-virus interactions, water deficit

\section{INTRODUCTION}

Under field conditions, plants are exposed to various biotic and abiotic stresses that can impact their performance and their population dynamics and ecology (Suzuki et al., 2014; Pandey et al., 2015; Prasch and Sonnewald, 2015; Ramegowda and Senthil-Kumar, 2015; Aou-ouad et al., 2017). In particular, soil water deficit (WD) and plant virus diseases are major abiotic and biotic constraints impacting plant physiology and growth as well as agricultural 
productivity worldwide (Zhang and Sonnewald, 2017). The relationships between host plants and viruses appear more complex than previously described, particularly regarding the role of perturbing environmental factors. To date, environmental conditions and plant physiological mechanisms that can lead to contrasted relationship with viruses, from mutualism to increased pathogenicity, remain poorly studied (Roossinck, 2015). Few recent investigations combining abiotic and biotic stresses clearly demonstrate that in addition to factors related to the virus and plant genotype the fate of plant-virus interactions also depends on the abiotic environment (Prasch and Sonnewald, 2013; Ramegowda and Senthil-Kumar, 2015; Fraile and GarcíaArenal, 2016; Aguilar et al., 2017; Carr, 2017). A growing body of data is uncovering the intimate entanglement of the plant physiological pathways involved in responses to various abiotic stresses and in defense against pathogens and herbivores (Bostock, 2005; Pandey et al., 2015; Nejat et al., 2016). For instance, antiviral plant immune responses may interact with responses to additional environmental changes through crosstalks among hormonal pathways (Alazem and Lin, 2015; Nejat et al., 2016).

On the one hand, it has been shown that heat, drought or salt stress enhance plant susceptibility to pathogens (Atkinson et al., 2013; Kissoudis et al., 2015). On the another hand, viruses can enhance the ability of plants to counteract abiotic stresses by inducing drought or cold tolerance (Xu et al., 2008; Hily et al., 2016). Although the mechanisms involved are not clear, the authors evoked the increase of potential osmoprotectants in virus-infected plants (Xu et al., 2008). In the case of Cucumber mosaic virus (CMV), drought tolerance in Arabidopsis thaliana is triggered by the $2 \mathrm{~b}$ viral RNA silencing suppressor protein, a viral protein interfering with abscisic acid-mediated plant signaling (Westwood et al., 2013). It has been speculated that this effect of $2 \mathrm{~b}$ may ultimately serve viruses by aiding host plants to survive periods of environmental stress (Westwood et al., 2013).

Virus transmission is a key epidemiological parameter for which most plant viruses rely on arthropods vectors (Bragard et al., 2013). Impacts of abiotic stresses on virus spread have long focused on the vector biology (e.g., developmental time, longevity, fecundity, migration) and ecology (Nancarrow et al., 2014; Davis et al., 2015). While most of these studies speculated on a possible impact of environmental changes on the rate of virus transmission, direct experimental support was only brought very recently (Dáder et al., 2016; Nachappa et al., 2016; van Munster et al., 2017; Yvon et al., 2017). Because some studies suggest that transmission should be positively correlated to virulence, due to their shared relationship with viral accumulation (Alizon et al., 2009; Froissart et al., 2010) and that the environment may change viral transmission rate, abiotic stresses may also affect the relationship between these epidemiological parameters. Unfortunately, the plant's surrounding environment is most often ignored when studying such epidemiological correlations (Fraile and García-Arenal, 2016).

In the present study, we monitored the effect of WD on various important viral life traits, such as viral accumulation, virulence and transmission. In order to explore the genetic variability of these traits and their relationships we selected nine wild accessions of A. thaliana (L.) Heynh (Brassicaceae) with known contrasted responses to WD (Rymaszewski et al., 2017). All accessions were infected with the Cauliflower mosaic virus (CaMV; Caulimoviridae), a non-circulative virus transmitted by aphids, and grown under strictly controlled environmental conditions in the high throughput phenotyping platform PHENOPSIS (Granier et al., 2006). Interestingly, our results suggest that the perturbing effects of WD on plant growth traits can change the trade-off between virus accumulation, virulence and transmission rate.

\section{MATERIALS AND METHODS}

\section{Plant Material and Growth Conditions}

We selected nine natural accessions of $A$. thaliana (Mr-0, Col0, Ct-1, Sha, Cvi-0, Mt-0, Bay-0, Ler-1 and Est-1) based on their contrasted responses to drought (Rymaszewski et al., 2017). Three to five seeds were sown at soil surface in $225 \mathrm{ml}$ pots filled with a 30:70 (v/v) mixture of clay and organic compost (Substrate SP 15\% KLASMANN). Soil water content was estimated for each pot before sowing, as previously described (Granier et al., 2006). Subsequent changes in pot weight were attributed to change in water status. Soil surface was moistened with a modified one-tenth strength Hoagland solution, and pots were placed in the PHENOPSIS growth chamber (Granier et al., 2006) in the dark for 2 days at $12^{\circ} \mathrm{C}$ air temperature and $70 \%$ air relative humidity. Pots were dampened with sprayed deionized water three times a day until germination. During germination phase (7 days), plants were cultivated under $8 \mathrm{~h}$ day length $\left(200 \mu \mathrm{mol} \mathrm{m} \mathrm{m}^{-2} \mathrm{~s}^{-1}\right.$ photosynthetic photon flux density, at plant height), air temperature was set to $20^{\circ} \mathrm{C}$, and air relative humidity was adjusted in order to maintain constant water vapor pressure deficit (VPD) at $0.6 \mathrm{kPa}$. Then, plants were thinned to one plant per pot and grown at $21 / 18^{\circ} \mathrm{C}$ day/night while VPD was set at $0.75 \mathrm{kPa}$. Each pot was daily weighed and watered with the modified Hoagland solution to reach the target soil relative water content $\left(\mathrm{RWC}_{\text {soil }}\right)$. $\mathrm{RWC}_{\text {soil }}$ was maintained at $1.6 \mathrm{~g} \mathrm{H}_{2} \mathrm{O} \mathrm{g}^{-1}$ dry soil until application of the WD treatment. One week before the application of the WD treatment, watering was done with deionized water until the end of the experiment (Figure 1A).

\section{Virus Purification and Plant Inoculation}

The CaMV isolate Cabb B-JI (Delseny and Hull, 1983) efficiently transmitted following a non-circulative strategy by the aphid species Myzus persicae, was used in this study. Virus particles were purified from CaMV-infected Brassica rapa cv. "Just Right" (turnip) plants according to Hull and Shepherd (1976). The quality and the quantity of purified virus were assessed by polyacrylamide gel electrophoresis under denaturing conditions (12\% SDS-PAGE) and by spectrometric measurements at 230, 260, and $280 \mathrm{~nm}$ (NanoDrop 2000 spectrophotometer). Virus concentration was estimated by spectrometry using the formula described by Hull and Shepherd (1976).

One-month-old $A$. thaliana plants (20 plants per accession per treatment) were mechanically inoculated as previously described 


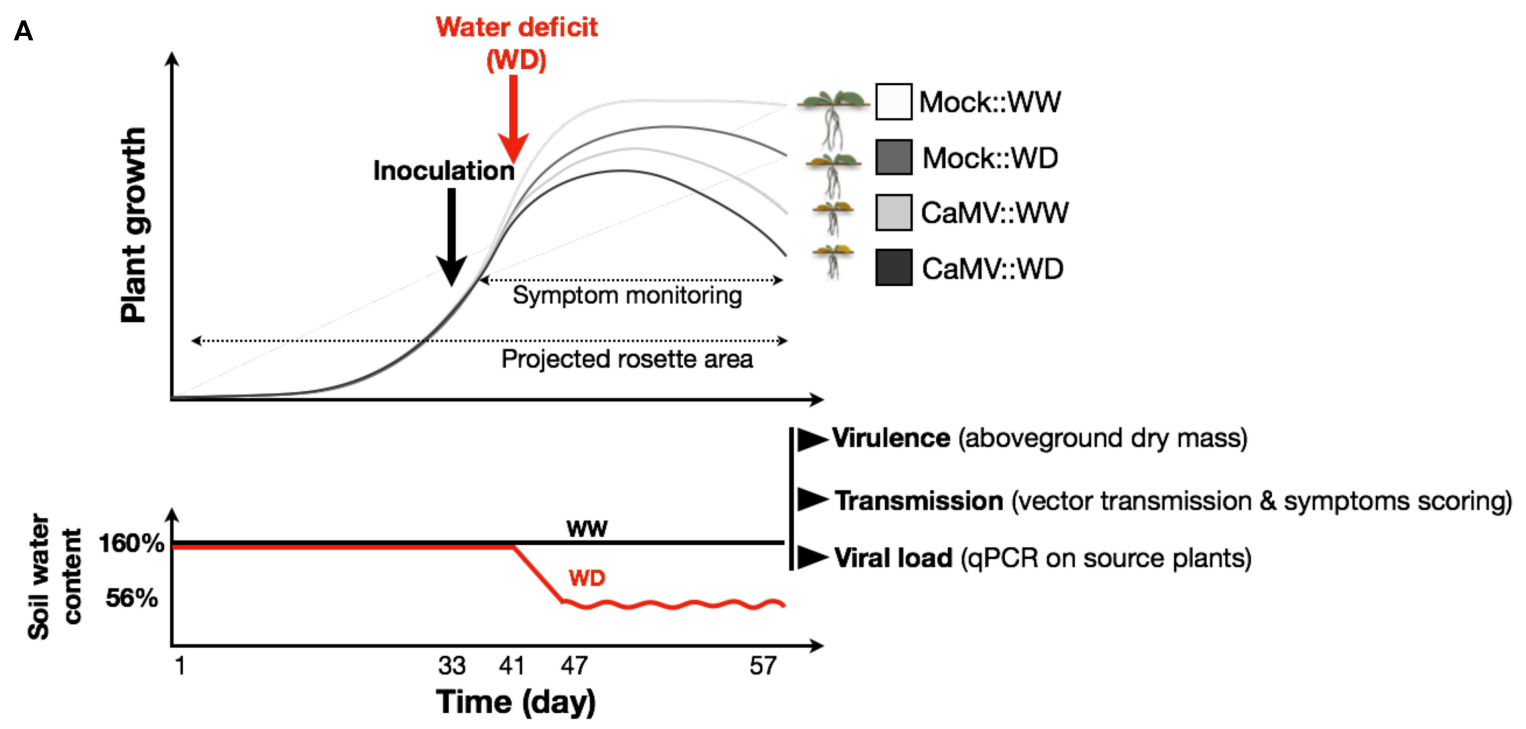

B

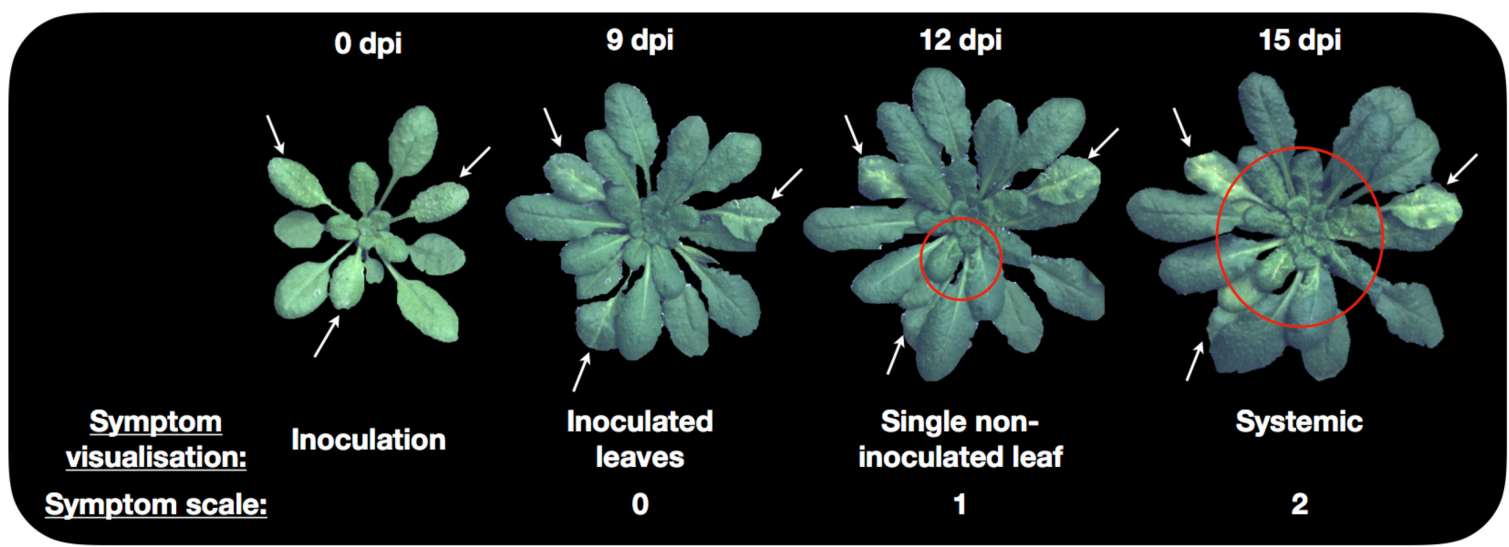

FIGURE 1 | Schematic representation of the experiment and timing of measurements. (A) Plant inoculation (mock- or CaMV; black arrow) was realized 33 days after germination and corresponds to the beginning of symptom scoring. One week later, soil water deficit treatment (WD; red arrow) was applied by stopping watering. Mean soil water content of individual pots during the course of the experiment is represented in the second panel. Soil relative water content was maintained at $160 \%$ in the control treatment (WW; black line) while stopping irrigation in the WD treatment brought the soil water content to $56 \%$ after 7 days and was maintained to this value by an adequate water supply (WD; red line). At the end of the experiment (day 57), viral traits (virulence, transmission efficiency and viral accumulation in source plants) were estimated. (B) Symptom monitoring was performed daily (from 9 to 25 dpi) on CaMV-infected:WW $(n=20)$ and CaMV-infected:WD $(n=20)$ plants. Symptoms were scaled 0, 1, or 2 for no symptom (symptom on inoculated leaves), presence of symptoms on a single non-inoculated leaf, or systemic symptoms, respectively.

(Blanc et al., 1993) with a mix containing CaMV-infected turnip extract enriched with the virus purification. Briefly, CaMVinfected turnip extract was prepared from $1 \mathrm{~g}$ of infected leaf material [leaves presenting systemic symptoms collected at 21 days post inoculation (dpi)] grinded in $1 \mathrm{ml}$ of distilled water. Purified CaMV particles were then added to this mix at a final concentration of $0.2 \mathrm{mg} \mathrm{ml}^{-1}$ to optimize infection success. For each inoculated plant, three leaves of median rank in the rosette were rubbed with a small pestle soaked in the solution described above. Control group (eight plants per accession and per watering treatment) was mock-inoculated in a similar way to mimic the wound induced by mechanical inoculation. Mock-inoculation was performed with a mix containing non-infected plant extract and the buffer used for virus purification $(100 \mathrm{mM}$ Tris- $\mathrm{HCl}$,
$2.5 \mathrm{mM} \mathrm{MgCl}_{2}, \mathrm{pH}$ 7). All plants were inoculated in a random order, independent of their accession and of the watering regime. In summary, four conditions representing mock-inoculated:WW $(n=8)$, mock-inoculated:WD $(n=8)$, CaMV-infected:WW $(n=20)$ and CaMV-infected:WD $(n=20)$ plants per accession were analyzed.

\section{Water Deficit Treatment}

One week after inoculation, corresponding to the approximate time of appearance of the first symptoms, irrigation of half of the CaMV- and mock-inoculated plants was stopped to reach the WD treatment at $0.56 \mathrm{H}_{2} \mathrm{O} \mathrm{g}^{-1}$ dry soil (this value was reached after 7 days of water deprivation). $\mathrm{RWC}_{\text {soil }}$ was then maintained to this value until the end of the experiment. In the well-watered 
treatment (WW) $\mathrm{RWC}_{\text {soil }}$ was maintained at $1.6 \mathrm{~g} \mathrm{H}_{2} \mathrm{O} \mathrm{g}^{-1}$ dry soil (Figure 1A).

\section{Measurement of Plant Traits and Symptoms Development}

Projected rosette area (7-10 plants per accession and treatment) was estimated from automated daily pictures using a semi-automatic procedure developed in the image analysis environment Image J (Research Services National Institute of Mental Health, Bethesda, MD, United States) and downloadable on the PHENOPSIS web site (Fabre et al., 2011). For each accession and watering treatment (WW and WD), four mock-inoculated and ten CaMV-infected individual plants were harvested $25 \mathrm{dpi}$ and individual aboveground dry mass was determined after 5 days at $60^{\circ} \mathrm{C}$.

Symptom monitoring was performed daily (from 9 to $25 \mathrm{dpi}$ ) on CaMV-infected:WW $(\mathrm{n}=20)$ and CaMV-infected:WD $(\mathrm{n}=20)$ plants for each accession. The symptoms were scaled 0,1 , or 2 for no symptom, presence of symptom on a single non-inoculated leaf, or systemic symptoms, respectively (see illustration on Figure 1B). Time of symptoms appearance, rate of systemic spread, and maximum proportion of infected plants were then calculated from logistic regressions fitted to these observations.

\section{Aphid Rearing}

The colony of the aphid-vector species $M$. persicae, collected over 30 years ago in the south of France was maintained on eggplants (Solanum melongena) in insect-proof cages, in a growth chamber at $23 / 18^{\circ} \mathrm{C}$ with a photoperiod of $14 / 10 \mathrm{~h}$ (day/night), in conditions ensuring clonal reproduction. Aphids were transferred to new cages and to new non-infested host plants (Solanum melongena) every 2 weeks, in order to avoid overcrowding and induction of the development of winged morphs.

\section{Aphid Transmission Assays}

Transmission efficiency of CaMV was assessed at $25 \mathrm{dpi}$ (Supplementary Figure S1). Batches of $20 \mathrm{M}$. persicae larvae (L2-L4 instars) were starved for $1 \mathrm{~h}$ before being transferred at the rosette center of a source plant for virus acquisition. Ten symptomatic source plants were used per accession and watering treatment. When aphids stopped walking and inserted their stylets into the leaf surface, they were allowed to feed for a short 2min period. Viruliferous aphids were then immediately collected in a Petri dish and individually transferred to 1-month-old Col-0 plantlets (test plants) grown under non-stressing conditions (one aphid per test plant; nine test plants per source plant). After an inoculation period of $3 \mathrm{~h}$, aphids were eliminated by insecticide spray $(0.2 \%$ Pirimor $\mathrm{G})$. Test plants were then placed in a growth chamber with the same conditions of air humidity, temperature and light as source plants and maintained under non-stressing conditions. Symptoms of virus infection were recorded 21 days later by visual inspection on test plants, as previously reported (Doumayrou et al., 2013; van Munster et al., 2017) and virus transmission rate was then calculated. After transmission assays, three leaves were randomly collected on each source plants and stored at $-80^{\circ} \mathrm{C}$ for further nucleic acid extraction and quantification of the virus accumulation.

\section{Plant DNA Extraction}

Total DNA from CaMV-infected leaf samples (pool of the three leaves collected per plant) was extracted according to a modified Edwards protocol (Edwards et al., 1991) with an additional washing step with $70 \%$ ethanol ( 10 biological replicates per accession and treatment). DNA was resuspended in $50 \mu \mathrm{l}$ of distilled water, and 10-fold dilutions were used as $\mathrm{qPCR}$ templates. The quality and quantity of the extracted total nucleic acid were assessed by spectroscopic measurements at 230, 260, and $280 \mathrm{~nm}$ (NanoDrop 2000 spectrophotometer).

\section{DNA Quantification by qPCR}

DNA quantification (10 biological replicates per accession and treatment, Supplementary Figure S1) was performed as duplicated $\mathrm{qPCR}$ in 384-well optical plates using the LightCycler FastStart DNA Master Plus SYBRGreen I kit (Roche) in a LightCycler 480 (Roche) thermocycler according to the manufacturer's instructions. Specific primers designed for the quantification of CaMV genome (Ca4443-F: 5'-GACCTA AAAGTCATCAAGCCCA-3' and Ca4557-R: 5'-TAGCTTT GTAGTTGACTACCATACG- $3^{\prime}$ ) and $A$. thaliana ubiquitinconjugating enzyme 21 gene (UBC21; UBC21_At_F: 5'-TGCA ACCTCCTCAAGTTCGA-3' and UBC21_At_R: $5^{\prime}$-GCAGG ACTCCAAGCATTCTT-3') were used at a final concentration of $0.3 \mu \mathrm{M}$. All qPCR reactions were performed with 40 cycles $\left(95^{\circ} \mathrm{C}\right.$ for $15 \mathrm{~s}, 62^{\circ} \mathrm{C}$ for $15 \mathrm{~s}$ and $72^{\circ} \mathrm{C}$ for $15 \mathrm{~s}$ ) after an initial step at $95^{\circ} \mathrm{C}$ for $10 \mathrm{~min}$. The $\mathrm{qPCR}$ data were analyzed with the LinReg PCR program to account for the efficiency of every single PCR reactions (Ruijter et al., 2009). The absolute initial viral concentration in A. thaliana plants, expressed in arbitrary fluorescence units ( $\mathrm{N}_{0} \mathrm{CaMV}$ ) was divided by that of $A$. thaliana UBC21 gene ( $\mathrm{N}_{0}$ UBC21; Genbank accession DQ027035), in order to normalize the amount of plant material analyzed in all samples.

\section{Data Analyses}

For each accession, the effects of the treatments on aboveground dry mass, transmission rate and viral load were analyzed by non-parametric Kruskal-Wallis tests. Time of symptoms appearance, rate of systemic spread, and maximum proportion of infected plants were extracted for each accession and watering treatment from logistic regression using the equation $\mathrm{A} /(1$ $\left.+\exp \left(\left(4^{*} \mu / \mathrm{A}\right) *(\lambda-\mathrm{t})+2\right)\right)$, where $\mathrm{A}$ is the maximum rate of infection, $\lambda$ is the time necessary for the appearance of a systemic symptom on a non-inoculated leaf and $\mu$ is the time necessary to detect systemic symptoms on the full plant. The effect of watering on transmission rate was tested in a generalized linear model (glm) model with the binomial link function. Response ratios of aboveground plant dry mass (the ratios of mean outcome in the experimental group to that in the control group) were used to quantify the response of each genotype to watering and viral infection (i.e., virulence). We tested the significance of the relationships between 
epidemiological parameters with the Spearman's rank correlation test.

All analyzes were performed in the programming environment R (R Core Team, 2017). Kruskal-Wallis tests were performed using the corresponding function in AGRICOLAE package. Bootstrapped $95 \%$ confidence intervals (CI) of mean trait values were computed following the mean_cl_boot procedure of the HMISC package. Non-linear models were fitted using the $n l s$ function and 95\% confidence intervals for the parameters of fitted models were computed with confint function of the package MASS. Generalized linear models were tested using the $g l m$ function of the STAT package. Mean response ratios and corresponding 95\% confidence intervals were calculated using sci.ratio of the MRATIO package.

\section{RESULTS}

\section{Systemic Spread Varies Between A. thaliana Accessions and, in Some Accessions, It Is Conditioned by Watering Treatment}

CaMV isolate Cabb B-JI successfully infected plants of all A. thaliana accessions selected. Symptoms, i.e., chlorotic lesions and vein-clearing of rosette leaves, were similar across accessions though their timing of appearance and intensity was greatly variable as detailed below. The proportion of CaMV-inoculated plants showing characteristic virus symptoms $25 \mathrm{dpi}$ varied from 96 to $100 \%$ across accessions whatever the soil watering treatment (Figure 2). In WW condition, the mean time of systemic symptoms appearance on the first non-inoculated leaf $( \pm 95 \%$ CI $)$ was $12.3( \pm 1.9)$ dpi (Figure 3A). However, lag time of systemic symptoms varied significantly between accessions from $10( \pm 0.3)$ dpi in Bay-0 to $14.7( \pm 0.2)$ dpi in Sha. Lag time did not change in response to WD in five accessions whereas it was significantly lower (i.e., faster appearance of firsts symptoms) in three accessions and higher in one accession (Figure 3A). For all accessions, the rate of systemic spread was lower under WD than in WW (Figure 3B). In particular, it was significantly reduced for Mr-0, Col-0, Ct-1, Sha and Cvi-0 (Figure 3B).

\section{Plant Growth Response to Water Deficit and Viral Infection Vary Across Natural Accessions}

We selected nine natural accessions of $A$. thaliana (Mr-0, Col0, Ct-1, Sha, Cvi-0, Mt-0, Bay-0, Ler-1 and Est-1) based on their contrasted responses to drought (Figure 4A). Plant aboveground dry mass $( \pm S D)$ ranged from $0.21( \pm 0.02) \mathrm{g}$ (Ler-1) to 0.46 $( \pm 0.04)$ g (Mr-0) under WW condition (Figure 4B). As determined 19 days after the start of the treatment, WD reduced aboveground dry mass production (20-25\% reduction) in the four accessions the less tolerant to WD: Mr-0, Col-0, Ct-1 and Sha. Growth reduction due to WD was marginally significant in Cvi-0 $(P=0.083)$, and not significant in Mt-0, Bay-0, Ler-1 and Est-1, which are accessions the most tolerant to WD (Figure 4B).
As determined at $25 \mathrm{dpi}$, CaMV virulence under WW, calculated as CaMV-infected:WW/mock-inoculated:WW for aboveground biomass, varied significantly among accessions. CaMV infection significantly reduced aboveground dry mass (10-21\% reduction) in all accessions but Ct-1, Sha and Est-1 (Figure 4B). In particular, aboveground dry mass of CaMVinfected plants was significantly reduced in Bay-0 $(P=0.0072)$, Col-0 $(P=0.048)$ and Mt-0 $(P=0.047)$.

In general, the combination of WD and viral infection tended to be more deleterious than each of the two stresses taken separately. However, plant responses differed widely between accessions. For example, WD and CaMV infection combination did not have a significant effect on aboveground mass of Est-1 and Ler-1 compared to the mock-inoculated:WW condition, whereas a $13-40 \%$ significant reduction was found in five other accessions $(P<0.05$; Figure 4B). However, in two accessions, Sha and Ct1 , stress combination was less severe than the effect of WD alone although it was no significant (Sha: $P=0.15$ and Ct-1: $P=0.20$; Figure 4B). Similar trends were observed for the projected rosette area since this trait was highly significantly correlated to aboveground dry mass $\left(R^{2}=0.68, P<0.001\right.$; Supplementary Figure S2). Response ratios of aboveground biomass of each accession and for the different combinations of treatment are presented in Supplementary Figure S3.

\section{Variation of Viral Load and Transmission Rate Under WD Is Dependent on the Accession}

Preliminary experiments showed that CaMV transmission rate (from a virus donor infected plant) did not vary significantly according to the identity of the accession used as test plant (receptor plant) in transmission experiments (data not shown). For practical reasons, we therefore used Col-0 as test plants in subsequent transmission assays. Whatever the watering treatment and accession, transmission rate varied from 30 to $57 \%$ (Figure 5A). Surprisingly, while in most accessions WD did not significantly affect the transmission rate or marginally reduced it (Ler-1, from 55 to $38 \%, P<0.10$ ), we observed a significant increase of transmission rate in $\mathrm{Mr}-0$ and Sha (Figure 5A). In these two latter accessions transmission rate increased from 35$51 \%$ to $38-57 \%$, respectively $(P<0.05)$. Noteworthy, a similar trend was observed for Ct-1 and Est-1 (Figure 5A).

Viral load, i.e., CaMV accumulation in source plants, significantly decreased when plants were grown under WD compared to WW condition for Mr-0, Ct-1, and Sha (10-25\% reduction, $P<0.05)$, and tended to decrease in Col-0, Cvi-0, Mt-0 and Ler-1 (Figure 5B). No correlation was found between viral load and transmission rate across accessions whatever the watering condition (Spearman's $r=0.40, P=0.29$ in WW; Spearman's $r=0.40, P=0.17$ in WD).

\section{Water Deficit Alters the Relationship Between Transmission Rate and Virulence}

A significant positive correlation between CaMV virulence (i.e., CaMV-infected:WW/mock-inoculated:WW response ratio 

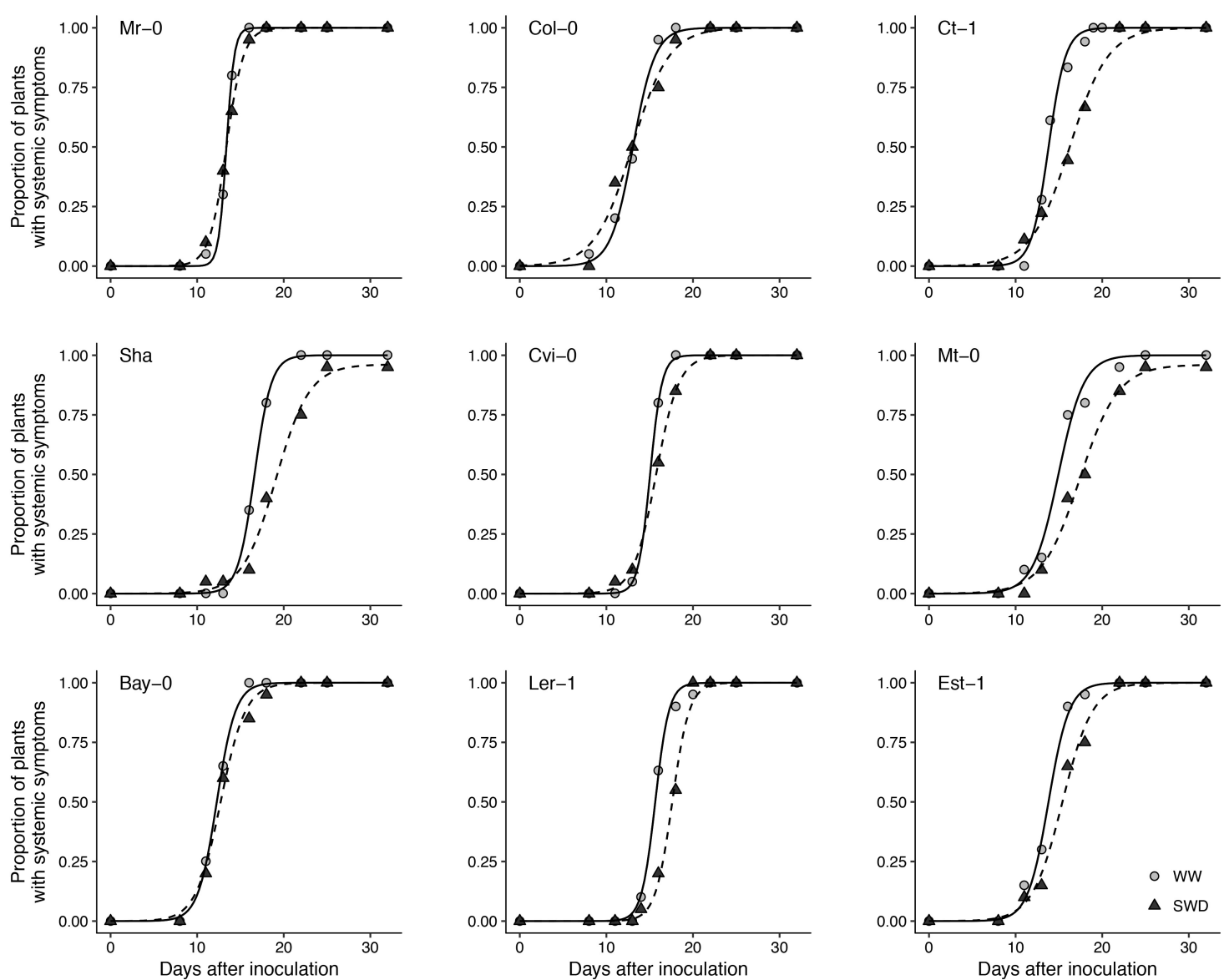

FIGURE 2 | Symptom dynamics in nine A. thaliana accessions inoculated with CaMV and grown under two watering conditions. Each panel represents one accession grown under well-watered (gray circles) and water deficit (black circles), respectively. Points are means of the proportion of plants with systemic symptoms $\left(n=20\right.$ plants per accession and watering treatment). Curves are logistic fitting following equation $\mathrm{A} /\left(1+\exp \left(\left(4^{*} \mu / \mathrm{A}\right)^{*}(\lambda-t)+2\right)\right)$, where $t$ is the number of days after inoculation, $\mathrm{A}$ is the maximum rate of infection, $\lambda$ is the time necessary for the appearance of symptoms on a non-inoculated leaf and $\mu$ is the time required to visualize systemic symptoms.

of aboveground dry mass) and transmission rate was found under WW (Spearman's $r=0.68, P<0.05$; Figure 6). In other words, transmission rate was higher in accessions the most susceptible to CaMV infection in terms of aboveground mass. Under WD, transmission rate increased in accessions that were the most tolerant to CaMV while the reverse trend was observed in accessions more susceptible to CaMV. As a result, a significant negative correlation between transmission rate under WD and CaMV virulence was found (Spearman's $r=-0.75, P<0.02$; Figure 6). The relationship between virulence and transmission was distorted regardless of the response ratio calculated for virulence (Supplementary Figures S4, S5).

\section{DISCUSSION}

We investigated plant responses to simultaneous exposure to WD and infection with one isolate of the CaMV in nine wild accessions of $A$. thaliana (L.) Heynh. We tested the hypothesis that perturbing effects of WD on plant growth traits would lead to changes in key epidemiological parameters such as systemic spread, virulence, viral load and transmission rate. Our results showed that under WD, viral infection spread into the plant was slower compared to WW treatment. Furthermore, WD had contrasted effects on CaMV transmission rate and viral load among $A$. thaliana accessions. Under WW, transmission rate tended to increase with the susceptibility of the accession to CaMV. Under WD, transmission rate and susceptibility were negatively correlated.

Most plant viruses move in a systemic way within the host plant (Matthews, 1991; Leisner et al., 1993). After inoculation by an aphid vector or by mechanical inoculation like in the present study, CaMV re-initiate infections in different and distant tissues, and such viral spread guarantees its survival (Schoelz et al., 2015). Virus spread within the host plant happens through cell-to-cell movement (via plasmodesmata) and long-distance movement mainly through phloem vessels (Matthews, 1991; Stavolone et al., 2005). It is known that the time at which viruses move out of the inoculated leaf into the rest of the plant varies widely depending on factors such as host and virus species, 

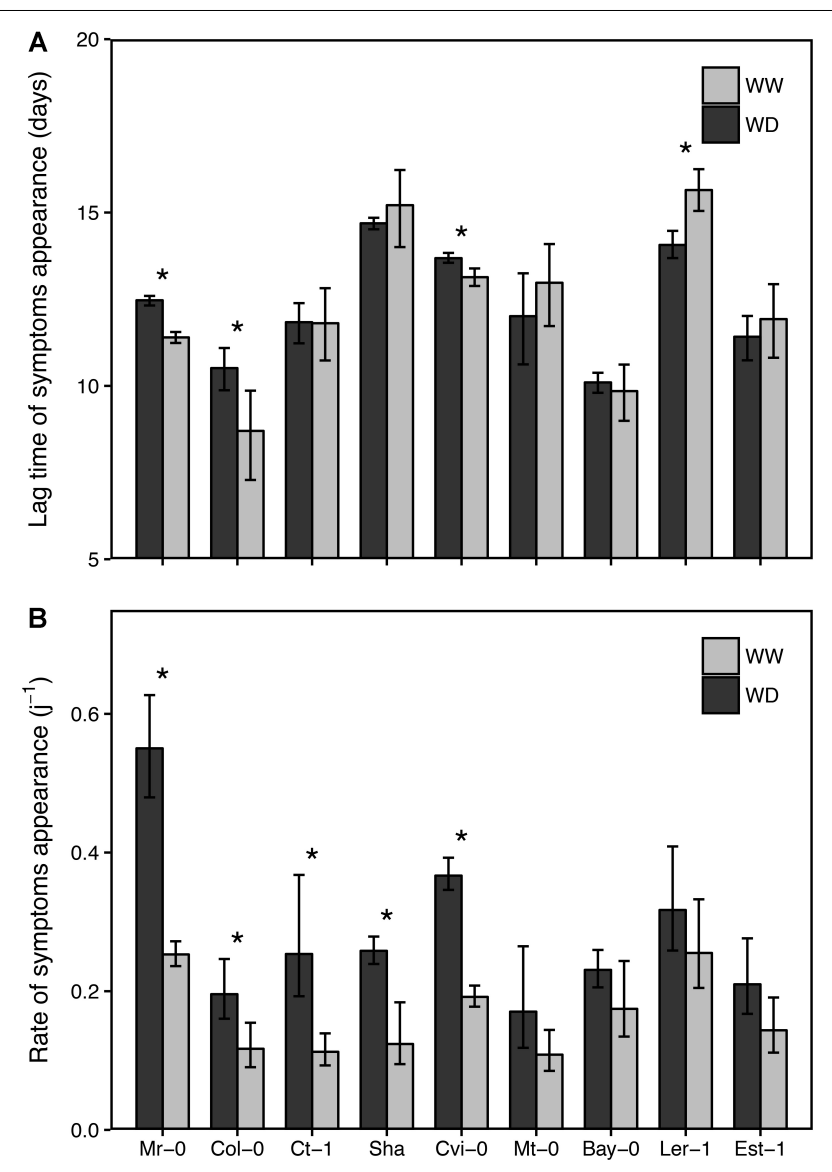

FIGURE 3 | Lag time and rate of CaMV symptoms appearance in nine A. thaliana accessions grown under two watering conditions. Bars and error bars are means and bootstrapped 95\% confidence intervals of lag time (A) and rate (B) of symptoms appearance extracted from sigmoidal curve fitting of symptom dynamics (see Figure 1) under well-watered (gray bars) and water deficit (black bars), respectively. Stars indicate significant difference between watering treatment for each accession (nonparametric Kruskal-Wallis tests; $P<0.05)$. Accessions are ordered according to increasing tolerance to WD.

age of the host, method of inoculation and abiotic constraints (Jensen, 1973; Ismail and Milner, 1988). In this study, we showed that the lag time to symptoms appearance (the mean time for symptoms to appear in the first non-inoculated leaf) and rate of systemic spread (the time required for all the plants of an accession to exhibit systemic symptoms) of CaMV was affected by accession identity and WD. Previous studies have already shown that inherent differences in development, particularly flowering phenology, and growth, can affect the dynamics of viral infection across accessions (Leisner et al., 1993). Virus spread is also influenced by the flow of metabolites in the plant (Bennett, 1940). Since WD may affect the relationship between carbon availability and sink organ growth (Muller et al., 2011), it can be assumed that long-distance transport of viral particles through phloem will also be affected, leading to slower systemic movement (Córdoba et al., 1991; Leisner et al., 1993; Leisner and Howell, 1993). Here, correlated with the negative effects of WD on plant growth and development we observed a significant reduction in the rate of systemic symptoms appearance in the most WD-sensitive accessions such as $\mathrm{Mr}-0$ and Ct-1. This reinforces the idea of a strong interaction between growth, phenology and viral infection dynamics. These observations are confirmed by the negative correlation between time or rate of systemic spread and tolerance to WD but also with the time of appearance of flowering buds (i.e., bolting) among accessions (not shown).

Regarding the impact of each independent stress on growth, CaMV infection had a generally lower negative effect on plant growth than WD since the virulence of the viral strain Cabb B-JI on vegetative growth was not too deleterious for most accessions studied. The effect of double stress was even more detrimental to rosette growth compared to control conditions. There was no correlation between tolerance to $\mathrm{WD}$ and tolerance to CaMV infection. Despite several reports on virus capacity to improve plant tolerance to abiotic stresses (Xu et al., 2008; Westwood et al., 2013), we could not find any significant positive effect of virus infection on plant tolerance to WD.

The success of transmission from infected to healthy host plants is crucial for the survival of all plant viruses. Interestingly, we showed that a WD triggered a significant increase of transmission rate in Sha and $\mathrm{Mr}-0$, two accessions among the most sensitive to WD as previously described (Rymaszewski et al., 2017). These results are consistent with the earlier report of increased transmission of CaMV and Turnip mosaic virus (TuMV) from B. rapa plants submitted to WD (van Munster et al., 2017). However, the variation of transmission rate under abiotic stress depends on the plant-virus-vector pathosystem and on the type of abiotic stress (Dáder et al., 2016; Nachappa et al., 2016; Yvon et al., 2017). It has been anticipated that abiotic stresses can impact multiple steps of the intricate plantvirus-vector interactions and so modify the transmission rates in many different ways (Mauck et al., 2012; Blanc and Michalakis, 2016). For example, it was demonstrated that the CaMV can 'sense' the aphid feeding activity and immediately produce transmissible morphs, and that this viral "behavior" is also triggered by some abiotic stresses (Martinière et al., 2013). This remarkable phenomenon has been designated 'transmission activation' (Martinière et al., 2013). It is most probably triggered by activation of plant defense pathways against aphid attacks, and that some abiotic stresses induce similar effects is likely due to partially overlapping pathways (Kiegle et al., 2000; Fujita et al., 2006; Suzuki et al., 2014; Pandey et al., 2015). In our study, we revealed that most WD-sensitive accessions have an increased CaMV transmission rate under WD, despite a reduced virus accumulation. This surprising observation might reflect a higher induction of signaling/defense pathways than in others accessions, and thus potentially, a stronger 'transmission activation' of the CaMV.

Virus accumulation in source plants was evaluated as a potential explanatory factor of the altered transmission efficiency. Indeed, it has been reported that abiotic stresses modify viral load of several viruses, such as Tobacco mosaic virus and Potato virus $A$, within stressed host plants (Dorokhov et al., 2012; Suntio and Mäkinen, 2012). Moreover, a positive correlation between virus load and transmission efficiency has also been reported in 
A

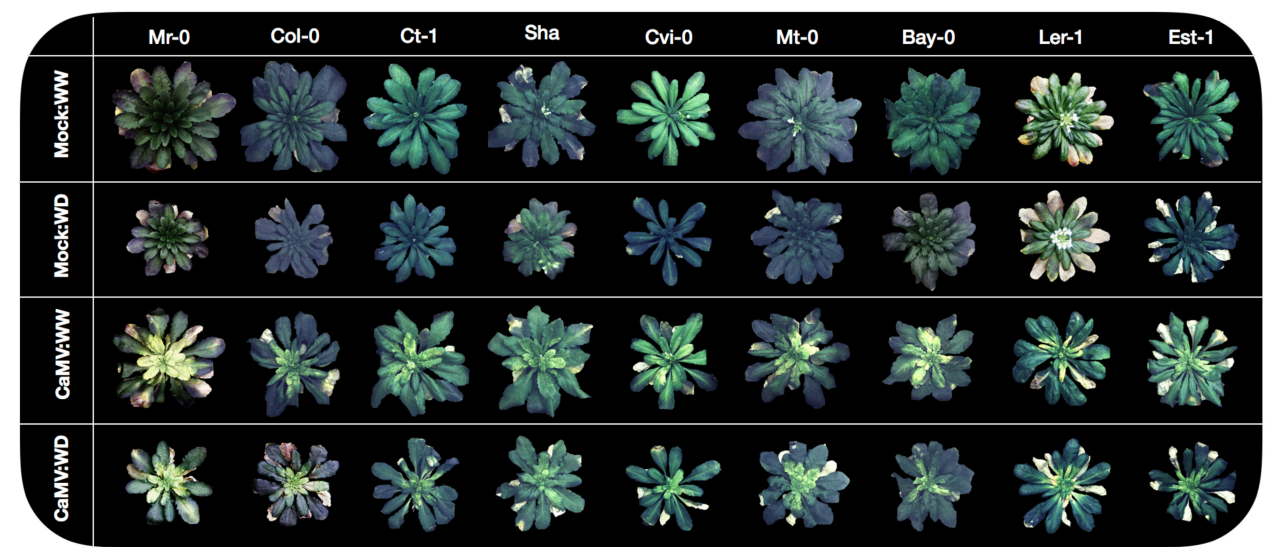

B

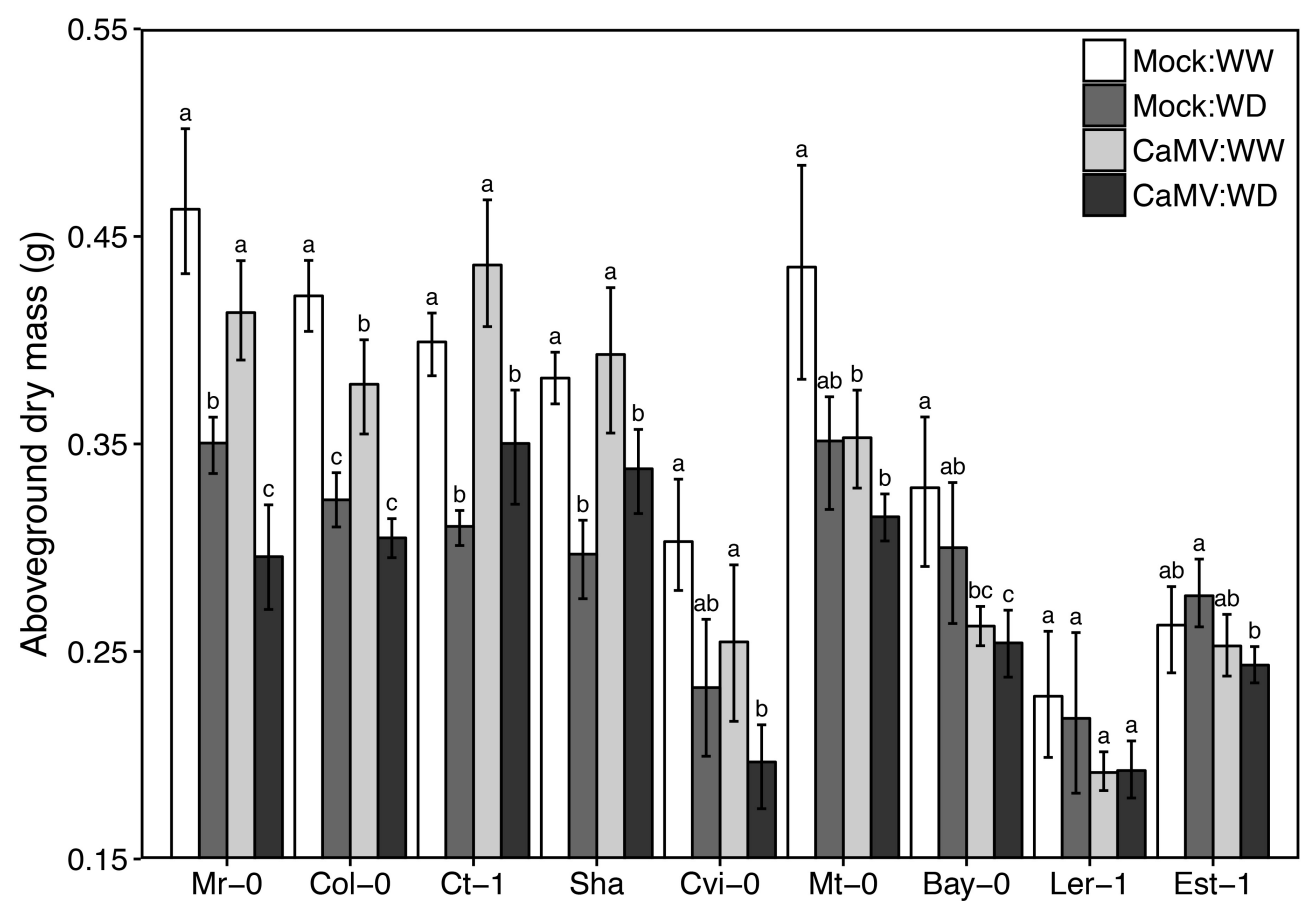

FIGURE 4 | Illustrative photographs of the rosettes and aboveground dry mass of nine $A$. thaliana accessions inoculated with CaMV and grown under two watering conditions. (A) Illustrative photographs of the rosettes of nine A. thaliana accessions (Mr-0, Col-0, Ct-1, Sha, Cvi-0, Mt-0, Bay-0, Ler-1 and Est-1) inoculated with a mock treatment or with CaMV and grown under well-watered (WW) or water deficit (WD) conditions. All photographs were taken 24 days postinoculation (dpi), 1 day before the transmission test. (B) Aboveground dry mass of nine A. thaliana accessions. Bars and error bars are means \pm bootstrapped $95 \%$ confidence intervals of aboveground dry mass 25 dpi and 19 days after the start of water deficit treatment (WD). Mock-inoculated:WW (white bars, $n=8$ ), mock-inoculated:WD (dark gray bars, $n=8$ ), CaMV-infected:WW (light gray bars, $n=20$ ) and CaMV-infected:WD (black bars, $n=20$ ) conditions. Different letters indicate a significant difference between treatments for each accession (nonparametric Kruskal-Wallis tests; $P<0.05$ ). Error bars were not used to interpret the results. Accessions are ordered according to increasing tolerance to WD.

some instances (Froissart et al., 2010; Doumayrou et al., 2013). In our study, we could not find any significant correlation between these two traits, i.e., a higher viral particles accumulation in the plant did not invariably correlate with a higher transmission. In addition to the phenomenon of the transmission activation discussed above, we also emphasize that many other unknown factors may be responsible for the observed altered transmission under water deficit condition. For example, feeding behavior of the aphid vector may differ on WD infected plants as a consequence of change in plant quality such as changes in carbonto-nitrogen ratio (Gutiérrez et al., 2013; Prasch and Sonnewald, 2013; Trębicki et al., 2016) and/or morpho-anatomical changes (Carmo-Sousa et al., 2014; Dáder et al., 2017).

In our CaMV-Arabidopsis pathosystem, virulence, as measured by the response ratio of aboveground dry mass, was significantly positively and linearly correlated to transmission rate under WW. The positive relationship between basal virulence and transmission is in accordance with evolutionary 

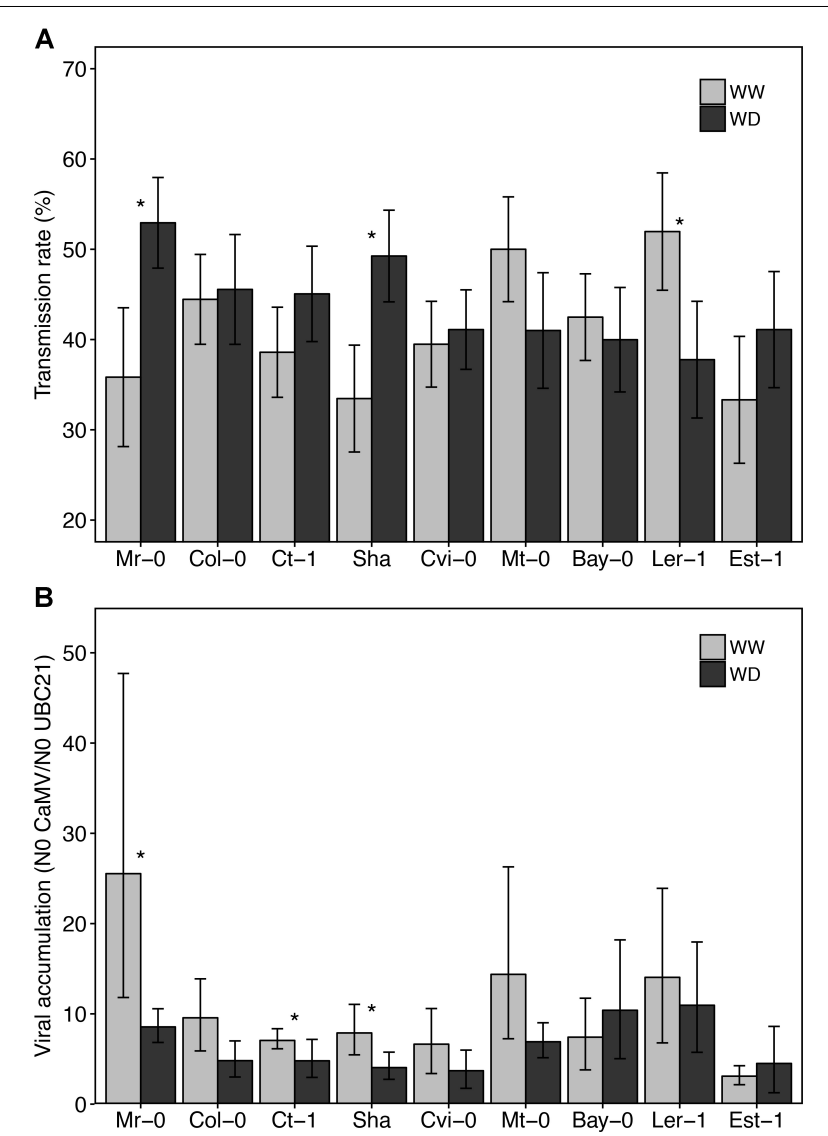

FIGURE 5 | CaMV transmission rate and accumulation in nine A. thaliana accessions grown under two watering conditions. Transmission rate (A), i.e., mean proportion of infected test plants ( $n=9$ per source plant) and viral load (B) of source plants $(n=10)$ of each accession grown under well-watered (gray bars) and water deficit (black bars), 25 dpi and 19 days after the start of water deficit treatment, respectively. Stars indicate a statistically significant difference between watering treatment for each accession (nonparametric Kruskal-Wallis tests; $P<0.05)$. Error bars represent bootstrapped 95\% confidence intervals but were not used to interpret the results. Accessions are ordered according to increasing tolerance to WD.

expectations as well as other empirical observations (Asplen et al., 2012; Doumayrou et al., 2013; Alizon and Michalakis, 2015). The 'trade-off hypothesis' postulates that increased virulence of a parasite is a viable evolutionary strategy if and only if its costs (mortality of the host) are counterbalanced by an increased transmission efficiency (Pagán et al., 2007). For instance, a positive relationship but with a saturating trend between these two traits was found in the CaMV-Brassica rapa pathosystem by Doumayrou et al. (2013) when testing different natural CaMV isolates on one cultivar of B. rapa. Surprisingly, we showed that WD had a significant reversing effect on this relationship. Indeed, CaMV transmission rate from the most virus-tolerant $A$. thaliana accessions (Sha, Ct-1, Est-1) significantly increased under WD while it decreased or did not change in other accessions. To our knowledge, this is the first study showing an alteration of the relationship (here reversed) between viral tradeoffs due to a change in abiotic conditions (Fraile and García-Arenal, 2016).

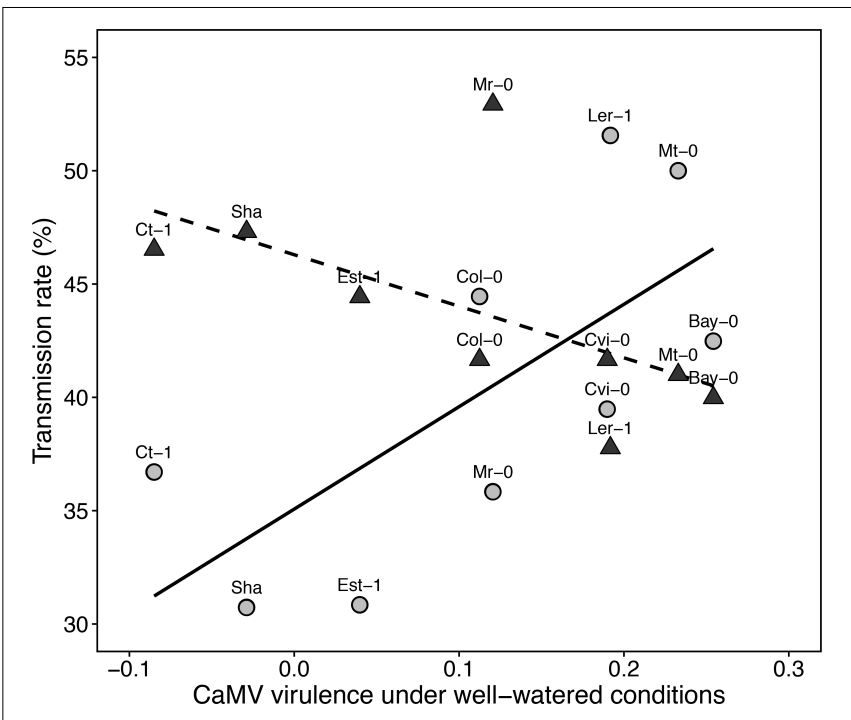

FIGURE 6 | Relationship between virulence and transmission rate of CaMV in nine $A$. thaliana accessions. Each labeled point represents CaMV virulence under well-watered and transmission rate under well-watered (gray circles) and water deficit (black triangles) for each accession. Lines represent linear regressions under well-watered (solid line; Spearman's $r=0.68, P=0.05$ ) and water deficit (dashed line; Spearman's $r=-0.75, P=0.019$ ), respectively.

\section{CONCLUSION}

Our results support the idea that optimal virulence of a given virus, as hypothesized under the transmission-virulence tradeoff, is highly dependent on the environment and growth traits of the host. The multi-faceted relationships between virulence, viral load and transmissibility according to the environmental conditions experienced by the host will require further theoretical and experimental investigations. In particular, investigations concerning the behavior of the aphid (alteration of its behavior by the environment) but also on the implication of plant and virus genetic diversity. Especially if these relationships have to be incorporated into models of virus epidemiology under scenarios of climate changes.

\section{AUTHOR CONTRIBUTIONS}

SEB, MvM, SB, and DV conceptualized the study. SEB, MvM, $\mathrm{MD}, \mathrm{AB}, \mathrm{CV}-\mathrm{R}, \mathrm{GR}, \mathrm{MY}$, and DV performed the experiments. SEB, MvM, and DV analyzed the data and wrote the original draft. All coauthors edited and reviewed the final version of the paper.

\section{FUNDING}

This work was supported by the European Union and the Region Languedoc-Roussillon "Chercheur d'Avenir" (FEDER FSE IEJ 2014-2020; Grant Project “APSEVIR” \#2015005464). 


\section{ACKNOWLEDGMENTS}

We thank G. Fleury, A. Pepey, and L. Kohan for the technical support and image analysis during the experiments and S. Leblaye and J.-L Macia for aphid rearing.

\section{REFERENCES}

Aguilar, E., Cutrona, C., del Toro, F. J., Vallarino, J. G., Osorio, S., PérezBueno, M. L., et al. (2017). Virulence determines beneficial trade-offs in the response of virus-infected plants to drought via induction of salicylic acid: trade-offs in virus-infected plants to drought. Plant Cell Environ. 40, 2909-2930. doi: $10.1111 /$ pce. 13028

Alazem, M., and Lin, N.-S. (2015). Roles of plant hormones in the regulation of host-virus interactions. Mol. Plant Pathol. 16, 529-540. doi: 10.1111/mpp. 12204

Alizon, S., Hurford, A., Mideo, N., and Van Baalen, M. (2009). Virulence evolution and the trade-off hypothesis: history, current state of affairs and the future. J. Evol. Biol. 22, 245-259. doi: 10.1111/j.1420-9101.2008.01658.x

Alizon, S., and Michalakis, Y. (2015). Adaptive virulence evolution: the good old fitness-based approach. Trends Ecol. Evol. 30, 248-254. doi: 10.1016/j.tree.2015. 02.009

Aou-ouad, H. E., Pou, A., Tomàs, M., Montero, R., Ribas-Carbo, M., Medrano, H., et al. (2017). Combined effect of virus infection and water stress on water flow and water economy in grapevines. Physiol. Plant 160, 171-184. doi: 10.1111/ ppl.12541

Asplen, M. K., Bruns, E., David, A. S., Denison, R. F., Epstein, B., Kaiser, M. C., et al. (2012). Do trade-offs have explanatory power for the evolution of organismal interactions? Evolution 66, 1297-1307. doi: 10.1111/j.1558-5646.2011. 01573.x

Atkinson, N. J., Lilley, C. J., and Urwin, P. E. (2013). Identification of genes involved in the response of Arabidopsis to simultaneous biotic and abiotic stresses. Plant Physiol. 162, 2028-2041. doi: 10.1104/pp.113.222372

Bennett, C. W. (1940). Relation of food translocation to movement of virus of Tobacco mosaic. J. Agric. Res. 60, 361-390.

Blanc, S., Cerutti, M., Usmany, M., Vlak, J. M., and Hull, R. (1993). Biological Activity of Cauliflower mosaic virus aphid transmission factor expressed in a heterologous system. Virology 192, 643-650. doi: 10.1006/viro.1993.1080

Blanc, S., and Michalakis, Y. (2016). Manipulation of hosts and vectors by plant viruses and impact of the environment. Curr. Opin. Insect Sci. 16, 36-43. doi: 10.1016/j.cois.2016.05.007

Bostock, R. M. (2005). Signal crosstalk and induced resistance: straddling the line between cost and benefit. Annu. Rev. Phytopathol. 43, 545-580. doi: 10.1146/ annurev.phyto.41.052002.095505

Bragard, C., Caciagli, P., Lemaire, O., Lopez-Moya, J. J., MacFarlane, S., Peters, D., et al. (2013). Status and prospects of plant virus control through interference with vector transmission. Annu. Rev. Phytopathol. 51, 177-201. doi: 10.1146/ annurev-phyto-082712-102346

Carmo-Sousa, M., Moreno, A., Garzo, E., and Fereres, A. (2014). A nonpersistently transmitted-virus induces a pull-push strategy in its aphid vector to optimize transmission and spread. Virus Res. 186, 38-46. doi: 10.1016/j.virusres. 2013.12.012

Carr, J. P. (2017). Exploring how viruses enhance plants' resilience to drought and the limits to this form of viral payback. Plant Cell Environ. 40, 2906-2908. doi: $10.1111 /$ pce. 13068

Córdoba, A. R., Taleisnik, E., Brunotto, M., and Racca, R. (1991). Mitigation of tomato spotted wilt virus infection and symptom expression by water stress. J. Phytopathol. 133, 255-263. doi: 10.1111/j.1439-0434.1991.tb00160.x

R Core Team (2017). R: A Language and Environment for Statistical Computing. Vienna: R Foundation for Statistical Computing.

Dáder, B., Fereres, A., Moreno, A., and Trêbicki, P. (2016). Elevated CO2 impacts bell pepper growth with consequences to Myzus persicae life history, feeding behaviour and virus transmission ability. Sci. Rep. 6:19120. doi: 10.1038/ srep 19120

Dáder, B., Then, C., Berthelot, E., Ducousso, M., Ng, J. C. K., and Drucker, M. (2017). Insect transmission of plant viruses: multilayered interactions optimize

\section{SUPPLEMENTARY MATERIAL}

The Supplementary Material for this article can be found online at: https://www.frontiersin.org/articles/10.3389/fpls.2018.00703/ full\#supplementary-material

viral propagation: virus-insect-plant interactions in transmission. Insect Sci. 24, 929-946. doi: 10.1111/1744-7917.12470

Davis, T. S., Bosque-Pérez, N. A., Foote, N. E., Magney, T., and Eigenbrode, S. D. (2015). Environmentally dependent host-pathogen and vector-pathogen interactions in the Barley yellow dwarf virus pathosystem. J. Appl. Ecol. 52, 1392-1401. doi: 10.1111/1365-2664.12484

Delseny, M., and Hull, R. (1983). Isolation and characterization of faithful and altered clones of the genomes of Cauliflower mosaic virus isolates Cabb B-JI, CM4-184, and Bari I. Plasmid 9, 31-41. doi: 10.1016/0147-619X(83)90029-X

Dorokhov, Y. L., Komarova, T. V., Petrunia, I. V., Frolova, O. Y., Pozdyshev, D. V., and Gleba, Y. Y. (2012). Airborne signals from a wounded leaf facilitate viral spreading and induce antibacterial resistance in neighboring plants. PLoS Pathog. 8:e1002640. doi: 10.1371/journal.ppat.1002640

Doumayrou, J., Avellan, A., Froissart, R., and Michalakis, Y. (2013). An experimental test of the transmission-virulence trade-off hypothesis in a plant virus. Evolution 67, 477-486. doi: 10.1111/j.1558-5646.2012.01780.x

Edwards, K., Johnstone, C., and Thompson, C. (1991). A simple and rapid method for the preparation of plant genomic DNA for PCR analysis. Nucleic Acids Res. 19:1349. doi: 10.1093/nar/19.6.1349

Fabre, J., Dauzat, M., Nègre, V., Wuyts, N., Tireau, A., Gennari, E., et al. (2011). PHENOPSIS DB: an information system for Arabidopsis thaliana phenotypic data in an environmental context. BMC Plant Biol. 11:77. doi: 10.1186/14712229-11-77

Fraile, A., and García-Arenal, F. (2016). Environment and evolution modulate plant virus pathogenesis. Curr. Opin. Virol. 17, 50-56. doi: 10.1016/j.coviro. 2016.01.008

Froissart, R., Doumayrou, J., Vuillaume, F., Alizon, S., and Michalakis, Y. (2010). The virulence-transmission trade-off in vector-borne plant viruses: a review of (non-)existing studies. Philos. Trans. R. Soc. B Biol. Sci. 365, 1907-1918. doi: $10.1098 /$ rstb.2010.0068

Fujita, M., Fujita, Y., Noutoshi, Y., Takahashi, F., Narusaka, Y., YamaguchiShinozaki, K., et al. (2006). Crosstalk between abiotic and biotic stress responses: a current view from the points of convergence in the stress signaling networks. Curr. Opin. Plant Biol. 9, 436-442. doi: 10.1016/j.pbi.2006. 05.014

Granier, C., Aguirrezabal, L., Chenu, K., Cookson, S. J., Dauzat, M., Hamard, P., et al. (2006). PHENOPSIS, an automated platform for reproducible phenotyping of plant responses to soil water deficit in Arabidopsis thaliana permitted the identification of an accession with low sensitivity to soil water deficit. New Phytol. 169, 623-635. doi: 10.1111/j.1469-8137.2005.01609.x

Gutiérrez, S., Michalakis, Y., Van Munster, M., and Blanc, S. (2013). Plant feeding by insect vectors can affect life cycle, population genetics and evolution of plant viruses. Funct. Ecol. 27, 610-622. doi: 10.1111/1365-2435.12070

Hily, J.-M., Poulicard, N., Mora, M.-Á., Pagán, I., and García-Arenal, F. (2016). Environment and host genotype determine the outcome of a plant-virus interaction: from antagonism to mutualism. New Phytol. 209, 812-822. doi: $10.1111 /$ nph.13631

Hull, R., and Shepherd, R. J. (1976). The coat proteins of Cauliflower mosaic virus. Virology 70, 217-220. doi: 10.1016/0042-6822(76)90257-9

Ismail, I. D., and Milner, J. J. (1988). Isolation of defective interfering particles of Sonchus yellow net virus from chronically infected plants. J. Gen. Virol. 69, 999-1006. doi: 10.1099/0022-1317-69-5-999

Jensen, S. G. (1973). Systemic movement of barley yellow dwarf virus in small grains. Phytopathology 63, 854-856. doi: 10.1094/Phyto-63-854

Kiegle, E., Moore, C. A., Haseloff, J., Tester, M. A., and Knight, M. R. (2000). Celltype-specific calcium responses to drought, salt and cold in the Arabidopsis root. Plant J. 23, 267-278. doi: 10.1046/j.1365-313x.2000.00786.x

Kissoudis, C., Chowdhury, R., van Heusden, S., van de Wiel, C., Finkers, R., Visser, R. G. F., et al. (2015). Combined biotic and abiotic stress resistance in tomato. Euphytica 202, 317-332. doi: 10.1007/s10681-015-1363-x 
Leisner, S. M., and Howell, S. H. (1993). Long-distance movement of viruses in plants. Trends Microbiol. 1, 314-317. doi: 10.1016/0966-842X(93)90009-G

Leisner, S. M., Turgeon, R., and Howell, S. H. (1993). Effects of host plant development and genetic determinants on the long-distance movement of Cauliflower mosaic virus in Arabidopsis. Plant Cell 5, 191-202. doi: 10.1105/ tpc.5.2.191

Martinière, A., Bak, A., Macia, J.-L., Lautredou, N., Gargani, D., Doumayrou, J., et al. (2013). A virus responds instantly to the presence of the vector on the host and forms transmission morphs. eLife 2:e00183. doi: 10.7554/eLife.00183

Matthews, C. (1991). Plant Virology. San Diego, CA: Academic Press.

Mauck, K., Bosque-Pérez, N. A., Eigenbrode, S. D., De Moraes, C. M., and Mescher, M. C. (2012). Transmission mechanisms shape pathogen effects on host-vector interactions: evidence from plant viruses. Funct. Ecol. 26, 1162-1175. doi: 10. 1111/j.1365-2435.2012.02026.x

Muller, B., Pantin, F., Génard, M., Turc, O., Freixes, S., Piques, M., et al. (2011). Water deficits uncouple growth from photosynthesis, increase $\mathrm{C}$ content, and modify the relationships between $\mathrm{C}$ and growth in sink organs. J. Exp. Bot. 62, 1715-1729. doi: 10.1093/jxb/erq438

Nachappa, P., Culkin, C. T., Saya, P. M., Han, J., and Nalam, V. J. (2016). Water stress modulates soybean aphid performance, feeding behavior, and virus transmission in soybean. Front. Plant Sci. 7:552. doi: 10.3389/fpls.2016.00552

Nancarrow, N., Constable, F. E., Finlay, K. J., Freeman, A. J., Rodoni, B. C., Trebicki, P., et al. (2014). The effect of elevated temperature on Barley yellow dwarf virus-PAV in wheat. Virus Res. 186, 97-103. doi: 10.1016/j.virusres.2013. 12.023

Nejat, N., Rookes, J., Mantri, N. L., and Cahill, D. M. (2016). Plantpathogen interactions: toward development of next-generation diseaseresistant plants. Crit. Rev. Biotechnol. 37, 229-237. doi: 10.3109/07388551.2015. 1134437

Pagán, I., Alonso-Blanco, C., and García-Arenal, F. (2007). The relationship of within-host multiplication and virulence in a plant-virus system. PLoS One 2:e786. doi: 10.1371/journal.pone.0000786

Pandey, P., Ramegowda, V., and Senthil-Kumar, M. (2015). Shared and unique responses of plants to multiple individual stresses and stress combinations: physiological and molecular mechanisms. Front. Plant Sci. 6:723. doi: 10.3389/ fpls.2015.00723

Prasch, C. M., and Sonnewald, U. (2013). Simultaneous application of heat, drought, and virus to Arabidopsis plants reveals significant shifts in signaling networks. Plant Physiol. 162, 1849-1866. doi: 10.1104/pp.113.221044

Prasch, C. M., and Sonnewald, U. (2015). Signaling events in plants: stress factors in combination change the picture. Environ. Exp. Bot. 114, 4-14. doi: 10.1016/j. envexpbot.2014.06.020

Ramegowda, V., and Senthil-Kumar, M. (2015). The interactive effects of simultaneous biotic and abiotic stresses on plants: mechanistic understanding from drought and pathogen combination. J. Plant Physiol. 176, 47-54. doi: 10.1016/j.jplph.2014.11.008

Roossinck, M. J. (2015). Plants, viruses and the environment: ecology and mutualism. Virology 479-480, 271-277. doi: 10.1016/j.virol.2015.03.041

Ruijter, J. M., Ramakers, C., Hoogaars, W. M., Karlen, Y., Bakker, O., van den Hoff, M. J., et al. (2009). Amplification efficiency: linking baseline and bias in the analysis of quantitative PCR data. Nucleic Acids Res. 37:e45. doi: 10.1093/nar/ gkp045

Rymaszewski, W., Granier, C., Vile, D., Dauzat, M., Hennig, J., Kamrowska, D., et al. (2017). Stress-response gene expression reflects morpho-physiological responses to water deficit. Plant Physiol. 174, 1913-1930. doi: 10.1104/pp.17. 00318

Schoelz, J. E., Angel, C. A., Nelson, R. S., and Leisner, S. M. (2015). A model for intracellular movement of Cauliflower mosaic virus: the concept of the mobile virion factory. J. Exp. Bot. 67, 2039-2048. doi: 10.1093/jxb/erv520

Stavolone, L., Villani, M. E., Leclerc, D., and Hohn, T. (2005). A coiled-coil interaction mediates Cauliflower mosaic virus cell-to-cell movement. Proc. Natl. Acad. Sci. U.S.A. 102, 6219-6224. doi: 10.1073/pnas.0407731102

Suntio, T., and Mäkinen, K. (2012). Abiotic stress responses promote Potato virus A infection in Nicotiana benthamiana: $\mathrm{Ca} 2+$ and Potato virus A infection. Mol. Plant Pathol. 13, 775-784. doi: 10.1111/j.1364-3703.2012.00786.x

Suzuki, N., Rivero, R. M., Shulaev, V., Blumwald, E., and Mittler, R. (2014). Abiotic and biotic stress combinations. New Phytol. 203, 32-43. doi: 10.1111/nph.12797

Trębicki, P., Vandegeer, R. K., Bosque-Pérez, N. A., Powell, K. S., Dader, B., Freeman, A. J., et al. (2016). Virus infection mediates the effects of elevated CO2 on plants and vectors. Sci. Rep. 6:22785. doi: 10.1038/srep22785

van Munster, M., Yvon, M., Vile, D., Dader, B., Fereres, A., and Blanc, S. (2017). Water deficit enhances the transmission of plant viruses by insect vectors. PLoS One 12:e0174398. doi: 10.1371/journal.pone.0174398

Westwood, J. H., Mccann, L., Naish, M., Dixon, H., Murphy, A. M., Stancombe, M. A., et al. (2013). A viral RNA silencing suppressor interferes with abscisic acid-mediated signalling and induces drought tolerance in Arabidopsis thaliana. Mol. Plant Pathol. 14, 158-170. doi: 10.1111/j.1364-3703.2012.00840.x

Xu, P., Chen, F., Mannas, J. P., Feldman, T., Sumner, L. W., and Roossinck, M. J. (2008). Virus infection improves drought tolerance. New Phytol. 180, 911-921. doi: 10.1111/j.1469-8137.2008.02627.x

Yvon, M., Vile, D., Brault, V., Blanc, S., and van Munster, M. (2017). Drought reduces transmission of Turnip yellows virus, an insect-vectored circulative virus. Virus Res. 241, 131-136. doi: 10.1016/j.virusres.2017.07.009

Zhang, H., and Sonnewald, U. (2017). Differences and commonalities of plant responses to single and combined stresses. Plant J. 90, 839-855. doi: 10.1111/ tpj. 13557

Conflict of Interest Statement: The authors declare that the research was conducted in the absence of any commercial or financial relationships that could be construed as a potential conflict of interest.

The reviewer AG and handling Editor declared their shared affiliation.

Copyright (C) 2018 Bergès, Vile, Vazquez-Rovere, Blanc, Yvon, Bédiée, Rolland, Dauzat and van Munster. This is an open-access article distributed under the terms of the Creative Commons Attribution License (CC BY). The use, distribution or reproduction in other forums is permitted, provided the original author(s) and the copyright owner are credited and that the original publication in this journal is cited, in accordance with accepted academic practice. No use, distribution or reproduction is permitted which does not comply with these terms. 